\title{
A SURVEY ON RECENT WORK IN HYBRID FSO/RF COMMUNICATION LINK
}

\section{NAVDEEP KAUR \& HARMEET SINGH}

Assistant Professor, Chandigarh University, Punjab, India

\begin{abstract}
With the worldwide demand for higher bandwidth and speed, Free Space Optics (FSO) has emerged as a promising field to meet the requirement. However, simple FSO system faces a drawback of loss of data when transmitted through turbulent atmosphere. The loss can be so severe that it may lead to complete blackout of the communication channel. To overcome this problem, Hybrid FSO/RF is emerging as a communication field that can acts as a potential backhaul network when FSO fails. When main link deteriorate due to rain, fog, snow, haze, natural calamity or human activity, then millimeter wavelength radio frequency link (i.e. Hybrid FSO/RF) is utilized to provide the information at the receiver. This system provides high bandwidth with unlicensed frequencies along with high speed communication of data. In this paper, a literature survey on recent work done by some renowned researchers around the world in the field of hybrid FSO/RF is carried out which may be useful for future research activities. Further, future scope in this field is added for ready reference of researchers.
\end{abstract}

KEYWORDS: Free Space Optics, Hybrid FSO/RF, Outage Probability, Bit-Error-Rate \& Signal-to-Noise Ratio

Received: Apr 09, 2019; Accepted: Apr 29, 2019; Published: May 20, 2019; Paper Id.: IJECIERDJUN20194

\section{INTRODUCTION}

Free space optics (FSO) are the communication system in which the information is sent from transmitter to receiver through the atmosphere as unguided medium. Generally, the type of communication is line-of-sight (LOS). The distance may vary from few meters to few kilometers in earth's atmosphere and can extend to thousands of kilometers in satellite to satellite communication [1]. FSO provides tremendous data rate upto 10Gbps as compared to optical fiber which used fiber as guided media. FSO has wide range of applications in video surveillance and monitoring, cellular systems, security, broadcasting and military systems [2]. It is marked as the fastest mode of communication, however, it may tackle a number of impairments while transferring of data through turbulent atmospheric channel. Because of air as link, FSO is affected by various impairments before reaching at receiver end. There may be geometric loss, atmospheric loss due to fog, haze, rain etc.; misalignment loss due to pointing errors and many more. Turbulence induced fading leads to degradation in performance metrics, such as, outage probability, bit error rate (BER) and signal-to-noise ratio (SNR) which may lead to reduction in the system performance[3].

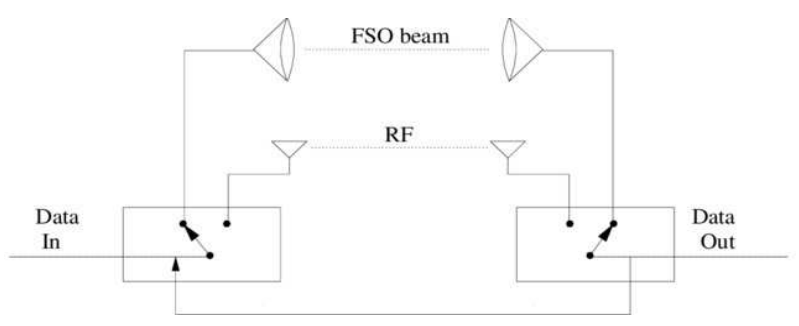

Figure 1: Schematic of Hybrid FSO/RF System [4] 
One of the recent remedy for the above mentioned degradations is Hybrid Free Space Optics /Radio Frequency (Hybrid FSO/RF) technique in which the FSO link acts as the primary communication channel and RF channel is used in case of high turbulence in atmosphere or in case the link gets destroyed due to natural calamity or human activity. Free space optical communication and millimeter wavelength radio frequency (FSO/RF) provides high capacity using unlicensed frequencies. The RF link provides backup to the communication system in case the FSO gets affected with different atmospheric conditions, such as, fog and rain causing high loss to data [5].

Hybrid FSO/RF has numerous applications like airborne, mobile communication and terrestrial applications because of its potential backup link. This paper is an attempt to review some of the recent research works done by some renowned researchers globally, which could be beneficial for advanced research in the field of hybrid FSO/RF communication. Apart from this, an attempt to discuss the future scope of research in this field is made in brief.

The rest of the paper is organized as follows: In section II literature survey on the research work of various researchers in the field of hybrid FSO/RF is done, section III provides the future scope, followed by conclusion in section IV and references in the end.

\section{LITERATURE SURVEY ON HYBRID FSO/RF}

The research work in the field of Hybrid FSO/RF by some globally prominent researchers in the period of 2015 to 2019 has been reviewed and presented as follows:

Tamer Rakia, et.al., (2015) analyzed hybrid FSO/RF communication system based on adaptive combing with a dual branch maximal ratio combiner at the receiver side. Commutative Distribution function expression is estimated and used to find the outage probability and transmission power. The results show that hybrid FSO/RF system with adaptive combining performs better in adverse atmospheric conditions than conventional one [6].

KuldeepKumaret, et al, (2015) presented quantize and encode relaying for the analysis of each bit with log likelihood ratio (LLR) into the symbol and at the receiver information is transmitted using the high speed FSO and hybrid FSO/RF link. The results show that relaying with FEC and without FEC code gives an improved gain using optimal symbol mapping [7].

Tamer, et.al., (2015) considered power adaptation strategies for hybrid FSO/RF system using adaptive combining. They proposed two strategies in which one depends on the received RF-SNR whereas the system is based on combined SNR of both links. The expressions for outage probability with and without adaptation are derived and the results show that system with power adaptation acquires higher outage performance as compared to the other [8].

Lei Kong, et. al., (2015) derived the closed form exact and asymptotic moment generating function in a dual hop. They calculated the average symbol error rate (ASER) of M- array phase shift keying (PSK), differential PSK (DPSK) and no coherent. Further, they evaluated ASER for K and Gamma-Gamma distributions, followed by ASER investigation for high SNR [9].

AbirTouatiet, et. al., (2015) presented a case study on the performance of hybrid SO/RF system on the harsh environment of region of Qatar. They modeled channel fading based on soft switching approach and a finite state Markove chains (FSMC) and derived the expression for outage probability. Further, they investigated the performance of the proposed system under hazy and harsh weather. FPGA based Raptor code interface was used to implement a soft switching 
algorithm and the results show that proposed algorithm renders lesser outage probability of hybrid system [10].

Vuong V. MAI, et. al., (2015) proposed hybrid FSO/RF system with adaptive multirate (AMR) concept for switching between two links of the system. Further, the cross layer design of AMR switching was presented using automatic repeat request (ARQ) scheme analytical framework using Markov Chain model was developed for system analysis using metrics of frame error rate, good put and link switching over fading channel. The results obtained verify the outperformance of the proposed system over the conventional one [11].

EhsanSoleimani -Nasab et al., (2015) analyzed mixed RF/ FSO system with generalized gamma turbulence with pointing error and Nakagami - $\mathrm{m}$ fading. Closed form expression for outage probability and bit error probability with Meijer's-G and two variable FOX-H functions. Further, they provided diversity gain analysis. Results show that outage and bit error rate probability is affected asymmetric as compared to symmetric interference [12].

MarziehNajafi et.al., (2016), worked on throughput maximization in parallel hybrid FSO/RF relay channel in which data was transmitted from source node to destination using multiple relays whereas nodes are orthogonal to each other. They derived the optimal relay selection and optimal time allocation policies and a gain is increased using proposed adaptive protocol as compared to conventional one [13].

AbirTouati, et.al., (2016) modelled closed form expression for bit error rate (BER) of FSO link with path loss, atmospheric and misalignment fading using on - off keying and Gamma-Gamma distribution. They calculated the effects of the combined fading on the outage probability for various strengths of turbulence and pointing errors with 16-Quardrature Amplitude Modulation (QAM) and Rician channel fading. The results show that degradation in link performance becomes prominent with pointing errors [14].

Saeede, et.al., (2016) proposed a relay based FSO system for backhaul purpose involving orthogonal frequency division multiple access setting. They proposed a resource allocation scheme to enhance the throughput in comparison to single FSO link in foggy conditions. The draw mentioned by them relates to the use of large number of relays which could be reduced to half the number [15].

Vahid, et. al., (2016) considered a cascade RF and Hybrid RF/FSO in which users transmit the data over RF link via relay node which is further forwarded to the destination through RF/FSO link. Fixed and adaptive link polices were derived for both delay limited and delay tolerant transmission. They considered DT-AL, DL-AL, DT-FL and DL-FL link polices to study the throughput for particular delay and signaling overheads. They proposed the future work as extension to the work done in this paper with other network architectures such as multi-hop serial / two hop parallel relay architecture [16].

BehroozMakki, et. al., (2016) analyzed the performance of hybrid FSO/RF links assuming perfect channel start information (CSI) at the receiver for with and without hybrid automatic repeat request (HARQ). They derived the closed from the expression for decoding probabilities, throughput and outage probability. Moreover the adaptive power allocation on throughput and outage probability were evaluated [17].

MarziehNajafi, et. al., (2016) tackled the problem of maximizing throughput in parallel hybrid RF/FQ relay channel. Relay system with and without buffer were studied and optimal relay selection and optimal time allocation policies were derived. Moreover, sub optimal buffer aided policy was purposed to introduce average delays [18]. 
Li chen, et.al., (2016) discussed multiuser diversity over parallel and hybrid FSO/RF. A hybrid FSO/RF point to multipoint system was proposed with link quality scheduling technique. An asymptotic closed form expression for outage probability and average bit error rate were analyzed [19].

Tamer, et.al., (2017) presented hybrid FSO/RF link analyzing point to multipoint (P2MP) network. They studied performance of system by metrics of throughput from central node to tagged node, transit buffer size, symbol delay, symbol loss probability and link utilization. The results shows that P2MP hybrid FSO/RF system performs better than simple PMP -FSO system [20].

KapalaVinodKiran, et. al., (2017) designed and experimentally verified hybrid FSO/RF system for communication between two computers under different channel conditions involving hard switching. ISM frequency band was used for RF communication link, which can give the motivational results for the current and future transmission system [21].

Yan Wil, et.al., (2017) considered dynamic link selection, power allocation and reliability guarantees for transmission of data in hybrid FSO/RF link to reduce the power consumption. Lyapunov optimization with notion of reliability queues was used to design dynamic link selection and power allocation (DLSPA) algorithm. The result shows that the power consumption can be reduced at the cost of reliability queue occupancy. The analytical results are verified through simulations [22].

Shubha, et. al., (2017) proposed a switching scheme in a dual hop scenario based on decode and forward (DF) relaying, where Nakagami-m and log normal distributions were considered for cumulative distribution function (CDF), Probability density function (PDF) and outage probability in closed from using Monte Carlo simulations. It is observed that the present system performs better than to variant parameter of FSO links as compared to severe fading of RF links [23].

U.A. Karai, et. al., (2017) represented a case study of the area of Hyderabad (Pakistan) where the effect of fog is negligible, however attenuation due to rain is significant. CCDF's of rain at both the links were calculated . The result shows that the desired data rate of hundreds of Gbps could be achieved with simple OOK modulation with link length less than a few hundred meters [24].

Shubha Sharma et.al., (2017), proposed a multiple- input-single-output (MISO) hybrid FSO/RF with transmit aperture/antenna selection (TAS) scheme. The proposed system was based on signal to noise ratio(SNR) if SNR is greater than threshold then FSO's best link is used whereas link of RF subsystem is used if SNR is less than threshold value. Outage probability and average symbol error rate (SER) of MISO and MISO hybrid FSO/RF were calculated [25].

Mohammad Ali Amirabadi, et. al., (2018) presented a relay assisted hybrid FSO/RF system in which a relay through RF link communicates with mobile users whereas a FSO link communicates with base station. The closed from expression for BER and outage probability were derived for fixed and adaptive gain relay schemes and verified through simulations. The results show that the fixed gain system involves less complexity, more power consumption and better performance [26].

Banibrata, et.al., (2018) proposed a multimeter wavelength (MMW)radio frequency FSO link used as backup in severe atmospheric conditions effecting the FSO link. They derived the mathematical expression for outage probability, average bit error rate(BER) and capacity. For irradiance fluctuation, Gamma- Gamma distribution is used followed by Rayleigh fading for multipath transmission [27]. 
M. Nasir Khan, et. al., (2018) developed a throughput maximization algorithm (TMA) for an adaptive FSO-radio frequency $(\mathrm{RF})$ communication system in which the performance is analyzed using regular and right regular low density parity check (LDPC) codes. The result shows that the TMA gives $2.25 \mathrm{db}$ higher gain in all weather conditions using right regular LDPC code [28].

Mohammad Ali Amirabadi, et. al., (2018) presented a dual hop relay assisted hybrid FSD/RF system in which an access point connected the users to the base station via the proposed system, which showed that the parallel combination of FSO and RF links along with the access point increased the capacity, reliability and the data rate. The FSO link was investigated using Gamma distribution with pointing errors and RF links was analyzed using Rayleigh fading. Closed form expression for bit error and outage probability was derived and verified using matlab simulation [29].

Maged A.Esmail, et. al.,(2019) experimentally analyzed the performance of all optical FSO link with $1 \mathrm{G}$ baud information using 16-Quadrature Amplitude Modulation (QAM) 5G signal under dusty environment . They demonstrated the results in terms of low visibility range, severe bit error rate and error vector magnilude (EVM) for $2.7 \mathrm{~m}$ channel length. The BER and EVM were improved upto $10^{-9}$ for $200 \mathrm{~m}$ of range. Further, the FSO and RF channels were compared for some dusty environment and the results obtained showed that RF link is almost negligible effected as compared to FSO link. To further improve system performance, hybrid cascaded FSO/RF link was installed [30].

ElyesBalti, et.al., (2019) presented a dual - hop RF/FSO system using multiple relays based on amplify and forward (AF) with fixed gain. Channel state information (CSI) was used with partial relay selection(PRS). Double Weibull fading was subjected by optical whereas Rayleigh fading was experienced by RF channel. For the reception either heterodyne or intensity modulation and direct detection (IM/DD) technique was used. The model of hardware impairments was introduced to source (S) and relays to quantify the effect of impairments. The closed form for high SNR asymptotic of outage probability (OP) and the ergodic capacity were derived using Monte-Carlo simulations [31].

\section{FUTURE SCOPE}

From the above mentioned work, it is evident that a large amount of work has been done in the recent past. However, there is always a scope to improve and a lot more can be further done in this field. Some of the ideas which can be incorporated are as follows:

- It is seen that very less work is done by introduction of pointing errors in Hybrid FSO/RF system. Therefore, pointing errors being an important metric to analyze the system performance should be introduced in a greater extent and different possibilities with this topic should be explored. Hence, it may contribute to a large scope in research in this area.

- $\quad$ Phase noise, being an important study area in FSO, also plays an important role in data transference. It gets added to the light signal as it passes through the turbulent channel and hence deteriorated the signal. To study the effect of phase noise on Hybrid FSO/RF signal, more work has to be done by the researchers.

- It is observed that very less number of the fading channel distributions have been used to study the system performances. The research work can be extended to combined channel distributions, developed by the combination of different channel models. 
- More recent modulation techniques can be introduced to study the effect of atmospheric turbulence on both (FSO and RF) links.

\section{CONCLUSIONS}

This paper is an attempt to review an emerging field in an optical communication system, i.e., Hybrid FSO/RF system. The literature survey in this field, for a period of 2015 to 2019 by eminent researchers across the globe, has been carried out and added in this paper. After reviewing this area of research, gaps have been found out and mentioned in section III for possible future work.

\section{REFERENCES}

1. Malik, A., \& Singh, P. (2015). Free space optics: current applications and future challenges. International Journal of Optics, 2015.

2. Khalighi, M. A., \& Uysal, M. (2014). Survey on free space optical communication: A communication theory perspective. IEEE communications surveys \& tutorials, 16(4), 2231-2258.

3. Ghassemlooy, Z., Popoola, W., \& Rajbhandari, S. (2017). Optical wireless communications: system and channel modelling with Matlabß. CRC press.

4. Usman, M., Yang, H. C., \& Alouini, M. S. (2014). Practical switching-based hybrid FSO/RF transmission and its performance analysis. IEEE Photonics Journal, 6(5), 1-13.

5. Ansari, I. S., Alouini, M. S., \& Yilmaz, F. (2013, April). On the performance of hybrid RF and RF/FSO fixed gain dual-hop transmission systems. In 2013 Saudi International Electronics, Communications and Photonics Conference (pp. 1-6). IEEE.

6. Rakia T., Yang HC, Alouini, M. S., \& Gebali, F. (2015),'”Outage analysis of practical FSO/RF hybrid system with adaptive combining, "' IEEE Communications Letters, 19(8), 1366-1369.

7. Kumar, K., \& Borah, D. K. (2015). Quantize and encode relaying through FSO and hybrid FSO/RF links. IEEE Transactions on Vehicular Technology, 64(6), 2361-2374.

8. Rakia, T., Yang, H. C., Gebali, F., \&Alouini, M. S. (2015). Power adaptation based on truncated channel inversion for hybrid FSO/RF transmission with adaptive combining. IEEE Photonics Journal, 7(4), 1-12.

9. Avadi, C. (2013). Subsequently Legion Optical Computer. Development (IJECIERD), 3(3), 53-64.

10. Kong, L., Xu, W., Hanzo, L., Zhang, H., \& Zhao, C. (2015). Performance of a free-space-optical relay-assisted hybrid RF/FSO system in generalized \$M \$-distributed channels. IEEE Photonics Journal, 7(5), 1-19.

11. Touati, A., Hussain, S. J., Touati, F., \& Bouallegue, A. (2015). Effect of atmospheric turbulence on hybrid FSO/RF link availability under Qatar harsh climate. Int. J. Electron. Commun. Eng., 9(8), 902-908.

12. Mai, V. V., \& Pham, A. T. (2015). Adaptive multi-rate designs and analysis for hybrid fso/rf systems over fading channels. IEICE Transactions on Communications, 98(8), 1660-1671.

13. Soleimani-Nasab, E., \& Uysal, M. (2015). Generalized performance analysis of mixed RF/FSO cooperative systems. IEEE Transactions on Wireless Communications, 15(1), 714-727.

14. Najafi, M., Jamali, V., \& Schober, R. (2016, May). Adaptive relay selection protocol for the parallel hybrid RF/FSO relay channel. In 2016 IEEE International Conference on Communications (ICC) (pp. 1-7). IEEE. 
15. Touati, A., Abdaoui, A., Touati, F., Uysal, M., \&Bouallegue, A. (2016). On the effects of combined atmospheric fading and misalignment on the hybrid FSO/RF transmission. Journal of Optical Communications and Networking, 8(10), 715-725.

16. Enayati, S., \&Saeedi, H. (2016). Deployment of hybrid FSO/RF links in backhaul of relay-based rural area cellular networks: Advantages and performance analysis. IEEE Communications Letters, 20(9), 1824-1827.

17. Jamali, V., Michalopoulos, D. S., Uysal, M., \&Schober, R. (2016). Link allocation for multiuser systems with hybrid RF/FSO backhaul: Delay-limited and delay-tolerant designs. IEEE transactions on wireless communications, 15(5), 3281-3295.

18. Barik, B. R., \& Kalirasu, A. Designing Of Trapezium Cut Shape Microstrip Antenna In X Band.

19. Makki, B., Svensson, T., Eriksson, T., \&Alouini, M. S. (2016). On the performance of RF-FSO links with and without hybrid ARQ. IEEE Transactions on Wireless Communications, 15(7), 4928-4943.

20. Najafi, M., Jamali, V., \&Schober, R. (2016). Optimal relay selection for the parallel hybrid RF/FSO relay channel: Non-buffer-aided and buffer-aided designs. IEEE Transactions on Communications, 65(7), 2794-2810.

21. Chen, L., Wang, W., \& Zhang, C. (2016). Multiuser diversity over parallel and hybrid FSO/RF links and its performance analysis. IEEE photonics journal, 8(3), 1-9.

22. Rakia, T., Gebali, F., Yang, H. C., \&Alouini, M. S. (2017). Cross layer analysis of P2MP hybrid FSO/RF network. Journal of Optical Communications and Networking, 9(3), 234-243.

23. Kiran, K. V., Rathore, S., Turuk, A. K., \& Das, S. K. (2017, October). Development of a Hybrid FSO/RF System during Link Misalignment. In 2017 International Conference on Networking and Network Applications (NaNA) (pp. 138-140). IEEE.

24. Wu, Y., Yang, Q., Park, D., \&Kwak, K. S. (2017). Dynamic Link Selection and Power Allocation With Reliability Guarantees for Hybrid FSO/RF Systems. IEEE Access, 5, 13654-13664.

25. Sharma, S., Madhukumar, A. S., Swaminathan, R., \& Sheng, C. J. (2017, December). Performance analysis of hybrid FSO/RF transmission for DF relaying system. In 2017 IEEE Globecom Workshops (GC Wkshps) (pp. 1-6). IEEE.

26. Korai, U. A., Luini, L., Nebuloni, R., \&Glesk, I. (2017, March). Statistics of attenuation due to rain affecting hybrid FSO/RF link: application for $5 G$ networks. In 2017 11th European Conference on Antennas and Propagation (EUCAP) (pp. 1789-1792). IEEE.

27. Sharma, S., Tan, J., Madhukumar, A. S., \&Swaminathan, R. (2017, December). Switching-Based Transmit Antenna/Aperture Selection in a MISO Hybrid FSO/RF System. In 2018 IEEE Global Communications Conference (GLOBECOM) (pp. 1-6). IEEE.

28. Sayehvand, J., \&Amirabadi, M. A. (2018). Performance analysis of hybrid FSO/RF communication systems with Alamouti Coding or Antenna Selection. arXiv preprint arXiv:1802.07286.

29. Adusumalli Mallikharjuna Rao, S. K. Overview Of Millimetre Wave Band To Be Used In $5 g$.

30. Bag, B., Das, A., Ansari, I. S., Prokě̌, A., Bose, C., \& Chandra, A. (2018). Performance analysis of hybrid FSO systems using FSO/RF-FSO link adaptation. IEEE Photonics Journal, 10(3), 1-17.

31. Khan, M. N., Gilani, S. O., Jamil, M., Rafay, A., Awais, Q., Khawaja, B. A.,\& Malik, A. W. (2018). Maximizing throughput of hybrid FSO-RF communication system: An algorithm. IEEE Access, 6, 30039-30048.

32. Amirabadi, M. A. (2018). Performance analysis of a novel hybrid FSO/RF communication system. arXiv preprint arXiv:1802.07160. 
33. Esmail, M. A., Ragheb, A. M., Fathallah, H. A., Altamimi, M., \&Alshebeili, S. A. (2019). 5G-28 GHz Signal Transmission over Hybrid All-Optical FSO/RF Link in Dusty Weather Conditions. IEEE Access, 7, 24404-24410.

34. Balti, E., Guizani, M., Hamdaoui, B., \&Maalej, Y. (2019, December). Partial relay selection for hybrid RF/FSO systems with hardware impairments. In 2016 IEEE Global Communications Conference (GLOBECOM) (pp. 1-6). IEEE. 